\title{
Improving the Event Data Server of the Fermilab Test Beam Data Acquisition System (OTSDAQ)
}

\author{
Emmanuel Noufele, Waubonsee Community College - CCI Program \\ Dr. Mandy Rominsky \& Dr. Evan Niner, Fermilab \\ FERMILAB-POSTER-19-077-PPD
}

\section{Description of the Fermilab Test Beam Facility}

The Fermilab Test Beam Facility (FTBF) is a high-energy test beam used for precision tests of high-energy physics detectors. The FTBF has two beams lines, MTest and MCenter, which provides a variety particle types such as proton beam and secondary beams with muons, electrons, and kaons. The beam is variable in energy and intensity, and a target can be inserted into the beam to convert the protons to other particle types for users.

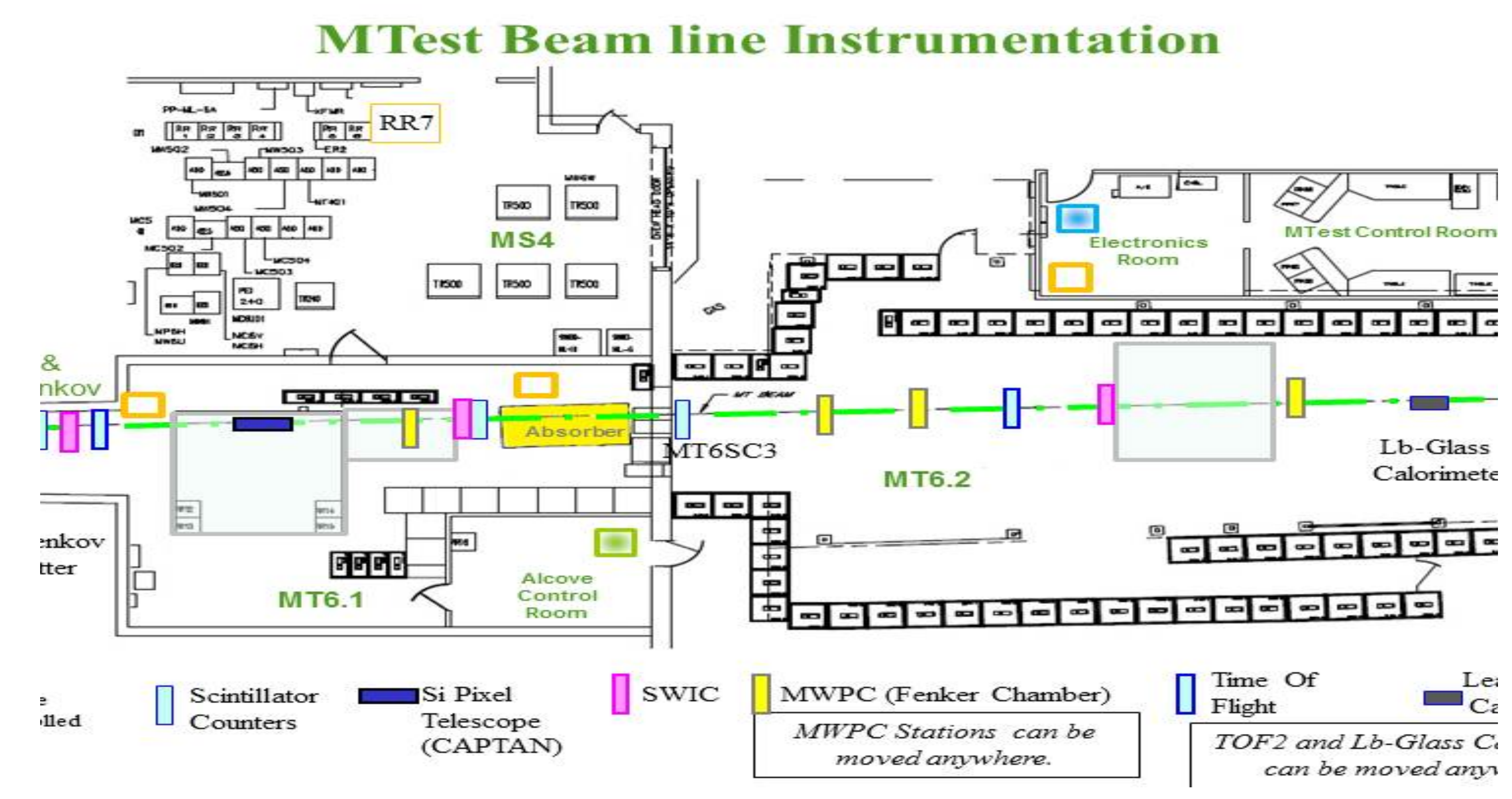

Figure 1 - Diagram of the FTBF beamline showing detector's places on the floor with control rooms for monitoring user's experimentation

\section{Description of the OTSDAQ}

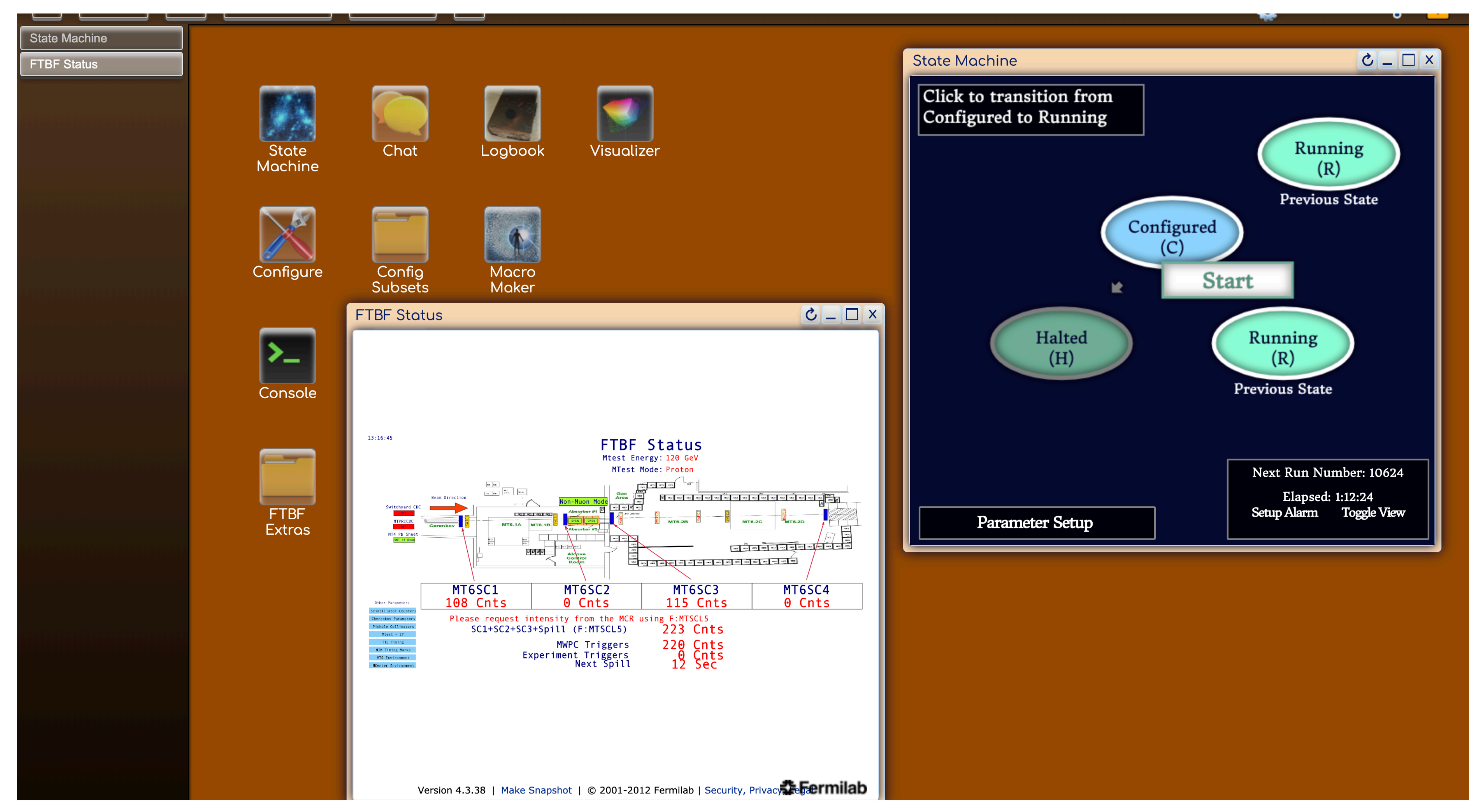

Figure 2 - The otsdaq Web Desktop

otsdaq is a "ready-to-use" Data Acquisition (DAQ) system, that needs just a small customization for simple installations. It was developed by the Fermilab Scientific Computing Division in order to fulfill the needs of the users. The figure above represents a web-based Run Control interface of the otsdaq that was developed for client. It has several features and tools that allows for automatically iterating over a parameter space to efficiently retrieve data and evaluate the detector response. My research project improved the Event Data Server framework that has not been run before and design a Transmission Control Protocol (TCP) socket for client in order to connect with the server.

\section{Methodology}

The otsdaq product was implemented as a Data Acquisition(DAQ) solution for the facility, initially reading out the telescope and the wire chambers. In addition to providing data to users, the system reads out all detectors on a spill-byspill basis. An Event Data Server has been developed to collect facility instrumentation data and stream it external user DAQ systems transparently. After editing, testing, and debugging the code using $\mathrm{C}++$, we were able to run and test the data server and client interface for the first time this summer as shown in figure 3.

\section{Results}

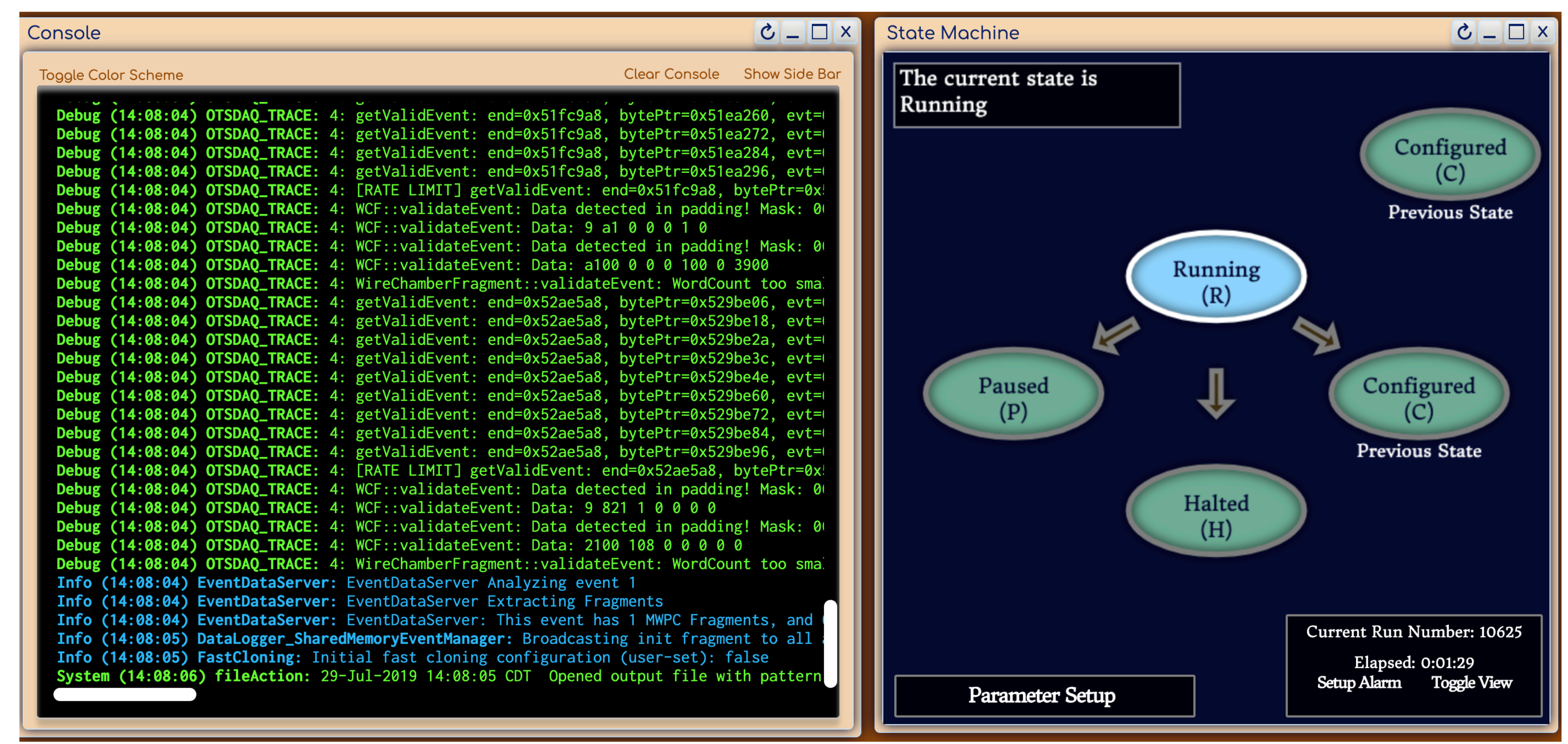

Figure 3 - The console is showing the connection of the Event Data Server with the otsdaq system

\section{Analysis and Conclusion}

The figure above shows how the console of the otsdaq interface looks like when the Event Data Server is analyzing and extracting fragments from the event received, which is a proof that the server is behave as it is suppose to. The next step is the design of the TCP socket client, which is underway. After that, users will be able to get data from other facility instruments in order to improve their detectors.

\section{Special thanks}

U.S Department of Energy - Community College Internships (CCI) Program for this opportunity.

Dr. Mandy Rominsky and Dr. Evan Niner (Summer mentors) for taking me on as their mentee and teaching me so many things in my short time here

Dr. Eric Flumerfelt for his time and help during my research

\section{References}

[1] "Fermilab Test Beam Facility," [online], https://ftbf.fnal.gov.

[2] Kurt Biery, Eric Flumerfelt, Adam Lyon, Ron Rechenmacher, Ryan Rivera, Mandy Rominsky, Lorenzo Uplegger, Margaret Votava, "The Fermilab Test Beam Facility Data Acquisition

System Based on otsdaq"[online], https://arxiv.org/pdf/1806.07240.pdf. 